DOI: 10.12731/2227-930X-2020-4-179-188

УДК 004.94

\title{
О СИСТЕМЕ ОЦЕНКИ КАЧЕСТВА РАБОТЫ ТРАНСПОРТНОГО ПРЕДПРИЯТИЯ ДЛЯ УПРАВЛЕНИЯ ЕГО ФУНКЦИОНИРОВАНИЕМ
}

Львович Я.Е., Преображенский А.П., Чопоров О.Н.

В статье рассматривается задача, связанная с оценкой качества транспортного предприятия. Дано описание модели управления. Указан критерий ее применения.

Ключевые слова: система перевозок; алгоритм; ресурс; управление.

\section{ABOUT THE SYSTEM \\ FOR ASSESSING THE QUALITY OF THE WORK OF THE TRANSPORTATION COMPANY FOR MANAGING ITS FUNCTIONING}

\section{Lvovich Ya.E., Preobrazhenskiy A.P., Choporov O.N.}

The paper deals with the problem associated with assessing the quality of a transport company. The description of the control model is given. The criterion for its application is indicated.

Ключевые слова: transportation system; algorithm; resource; control.

\section{Введение}

Необходимо на практике учитывать особенности фундаментальных процессов при функционировании систем транспортных предприятий, которые связаны с последовательным применением разных ресурсов $[1,2]$.

Есть возможности для того, чтобы определять базовые объекты оценок и управления в виде формирующихся структур качества 
транспортных систем, которые работают в рамках определенного $q$-го направления.

\section{Модель управления транспортной системой}

Ниже приведена общая модель. На ее основе происходит описание транспортной системы с точки зрения ее оценки и управлении. Что позволяет реализовать такая модель?

Есть возможности для того, чтобы определять связи среди той структуры качества транспортной системы, которая будет создаваться относительно $i$-го работника по $q$-му направлению $-Y_{q}^{(i)}$, а также целями его работы - $\bigsqcup_{q}^{(i)}$ и множеством средств, обеспечивающих возможности достижению подобных целей $-X_{q}^{(i)}$ :

здесь:

$$
\left\{\begin{array}{l}
Y_{l q}^{(i)}=F_{l q}^{(i)}\left(Y_{(l-1) q}^{(i)}, L_{l q}^{(i)}, X_{l q}^{(i)}, \omega_{l q}\right), \\
Y_{l q}^{(i)}=\left\{Y_{I l q}^{(i)}, Y_{I I q}^{(i)}\right\}, \\
Y_{I l q}^{(i)}=F_{I l q}^{(i)}\left(Y_{I(l-1) q}^{(i)}, Y_{I I(l-1) q}^{(i)}, L_{l q}^{(i)}, X_{l q}^{(i)}, W_{l q}\right), \\
Y_{I I l q}^{(i)}-F_{I I l q}^{(i)}\left(Y_{I I(l-1) q}^{(i)}, Y_{I(l-1) q}^{(i)}, L_{l q}^{(i)}, X_{l q}^{(i)}, Q_{l q}\right), \\
Y_{I l q}^{(i)}=\left\{Y_{I m l q}^{(i)}, m=\overline{1,4}\right\}, \\
Y_{\operatorname{Im} l q}^{(i)}=\left\{K_{m s l q}^{(i)}, K_{m \alpha l q}^{(i)}, K_{m \beta l q}^{(i)}, K_{m \tau l q}^{(i)}\right\}, \\
Y_{l q}^{(i)}=\left\{Y_{I I n l q}^{(i)}, \quad n=\overline{1, N}\right\}, \quad Y_{I I n l q}^{(i)}=\left\{K_{n r l q}^{(i)}\right\},
\end{array}\right.
$$

$l$ - этап работы сотрудника в транспортной компании $\left(l=\overline{l_{0}, l_{B}}, l_{0}-\right.$ начальный шаг, $l_{B}$ - конечный шаг; $m$ и $n$ - показывают обозначения компонентов, которые относятся к структуре качества в транспортной системе; $Y_{I(l-1) q}^{(i)}, Y_{I l q}^{(i)}-$ множество, которое сформировано фундаментальными знаниями и интеллектуальными умениями. В нем есть основная часть $Y_{l q}^{(i)}$ до и после $l$-го шага функционирования $i$-го работника; $Y_{I I(l-1) q}^{(i)}, Y_{I l q}^{(i)}-$ множество, которое сформировано при помощи знаний и умений, являющихся базовыми. В нем есть часть $Y_{l q}^{(i)}$ до и после $l$-го шага работы $i$-го сотрудника; $K^{(i)}-$ показатели того, насколько сформированы разные составляющие для структуры качества транспортной системы по $i$-му сотруднику $[3,4] ; \omega, \mathrm{W}$ и $\mathrm{Q}$ - представляют собой случайные факторы. 
Таблицุа 1.

\section{Иллюстрация компонентов в модели (1) относительно объекта, который касается оценок и управления}

\begin{tabular}{|c|c|}
\hline Обозначение & Описание содержательных характеристик \\
\hline 1 & 2 \\
\hline$Y_{I(l-1) q}^{(i)}, Y_{I l q}^{(i)}$ & $\begin{array}{l}\text { Иллюстрация множества, в которое входят четыре }(\mathrm{m}=4) \\
\text { свойства, которые являются интегральными. Формируется } \\
\text { оно при помощи знаний и умений } i \text {-го сотрудника в нача- } \\
\text { ле и конце } l \text {-го шага действий, когда рассматривается } q \text {-е } \\
\text { направление; }\end{array}$ \\
\hline$Y_{I I(l-1) q}^{(i)}, Y_{I I l q}^{(i)}$ & $\begin{array}{l}\text { Иллюстрация множества, которое формируется при помощи } \\
\text { знаний и умений } i \text {-го сотрудника в начале и конце } l \text {-го шага } \\
\text { действий, когда рассматривается } q \text {-е направление; }\end{array}$ \\
\hline $\begin{array}{l}Y_{\mathrm{Im} /}^{(i)}, \\
\text { при } \mathrm{m}=1, l=l_{\mathrm{B}} \\
l_{\text {в }}-\text { конечный } \\
\text { Этап работы }\end{array}$ & $\begin{array}{l}\text { сформированность у } i \text {-го сотрудника - картины работы } \\
\text { транспортного предприятия; }\end{array}$ \\
\hline $\begin{array}{l}Y_{\operatorname{Im} l q}^{(i)}, \\
\text { при } \mathrm{m}=2, l=l_{\mathrm{B}}\end{array}$ & $\begin{array}{l}\text { сформированность у } i \text {-го сотрудника системных } \\
\text { представлений о структуре производства, когда } \\
\text { рассматривается } q \text {-е направление; }\end{array}$ \\
\hline $\begin{array}{l}Y_{\operatorname{Im} l q}^{(i)} \\
\text { при } \mathrm{m}=3, l=l_{\mathrm{B}}\end{array}$ & $\begin{array}{l}\text { сформированность у } i \text {-го сотрудника представлений о пер- } \\
\text { спективах работы, когда рассматривается } q \text {-е направление; }\end{array}$ \\
\hline $\begin{array}{l}Y_{\operatorname{lm} l q}^{(i)}, \\
\text { при } \mathrm{m}=4, l=l_{\mathrm{B}}\end{array}$ & $\begin{array}{l}\text { сформированность у } i \text {-го сотрудника структуры } \\
\text { фундаментальных научных знаний, когда рассматривается } \\
q \text {-е направление; }\end{array}$ \\
\hline$K_{m s l q}^{(i)}$ & $\begin{array}{l}\text { Степень использования } i \text {-м сотрудником } m \text {-й компоненты } \\
\text { в } Y_{\mathrm{Il}} \text { после } l \text {-го этапа работы, когда рассматривается } q \text {-е } \\
\text { направление; }\end{array}$ \\
\hline$K_{\text {malq }}^{(i)}$ & $\begin{array}{l}\text { Степень использования } i \text {-м сотрудником } m \text {-й } \\
\text { компоненты в } Y_{\mathrm{Il}} \text { после } l \text {-го этапа работы, когда } \\
\text { рассматривается } q \text {-е направление; } \\
K_{m \alpha l q}^{(i)} \in \Omega_{\overline{k \alpha}}-\text { конечное множество уровней использования; }\end{array}$ \\
\hline$K_{m \beta l q}^{(i)}$ & $\begin{array}{l}\text { степень научности использования } i \text {-м сотрудником } m \text {-й ком- } \\
\text { поненты в } Y_{\mathrm{I} l} \text { после } l \text {-го этапа работы, когда рассматривается } \\
q \text {-е направление; }\end{array}$ \\
\hline$K_{m \tau l q}^{(i)}$ & $\begin{array}{l}\text { степень автоматизма навыков использования } i \text {-м сотруд- } \\
\text { ником } m \text {-й компоненты в } Y_{\mathrm{I} l} \text { после } l \text {-го этапа работы, когда } \\
\text { рассматривается } q \text {-е направление; }\end{array}$ \\
\hline$Y_{\text {IInl }}^{(i)}, \mathrm{n}=\overline{1, N}$ & $\begin{array}{l}\text { Множество умений и свойств } i \text {-го сотрудника, которые } \\
\text { связаны с тем, насколько быстро происходит восприятие } \\
\text { информации, ее обработка, синтез, установка взаимосвязей }\end{array}$ \\
\hline$K_{n}$ & $\begin{array}{l}\text { У } i \text {-го сотрудника степень развития } n \text {-го умения после } l \text {-го } \\
\text { этапа работы; }\end{array}$ \\
\hline
\end{tabular}


Можно провести переход от базового процесса управления (БПУ) (1) к обобщенному процессу управления (ОПУ). Тогда необходимо вести анализ по тому, какая общая качественная структура $[5,6]$ по всем сотрудникам в рамках $q$-го направления: $i=\overline{1, I}-Y_{q}^{(I)}$ :

$$
\left\{\begin{array}{l}
Y_{l q}^{(I)}=\left(F_{(l-) q}^{(I)}, L_{l q}^{(I)}, X_{l q}^{(I)}, \omega_{l q}\right) \\
Y_{l q}^{(I)}=\left\{Y_{l q}^{(I)}, Y_{l q}^{\prime \prime(I)}\right\} ; \quad Y_{l q}^{(I)}=\left\{1 / N_{I} * \sum_{i \in \Omega_{I}} Y_{l q}^{(i)}\right\} \\
Y_{l q}^{\prime(I)}=1 /\left(N_{I}-1\right) * \sum_{i \in \Omega_{I}}\left(Y_{l q}^{(i)}-Y_{l q}^{(I)}\right) \\
\ldots \ldots \ldots \ldots \ldots \ldots \ldots \ldots \ldots \ldots \ldots \ldots \ldots \ldots \ldots \ldots \ldots \ldots \ldots \ldots \ldots \ldots \ldots \ldots \ldots \ldots \ldots \ldots \ldots \ldots \ldots \ldots
\end{array}\right.
$$

При этом важно отметить, что нет изменений по общему виду модели в БПУ (1). Тогда не один ( $i$-й) сотрудник описывается переменными. На их базе идет формирование «коллективного портрета» по всему множеству сотрудников.

Исходя из того, какая структура БПУ, есть возможности для формирования структуры целей работы $[7,8]$. Ее можно рассматривать в виде качественной структуры в транспортной компании i-го сотрудника в рамках q-го направления, когда рассматривается l-й этап работы

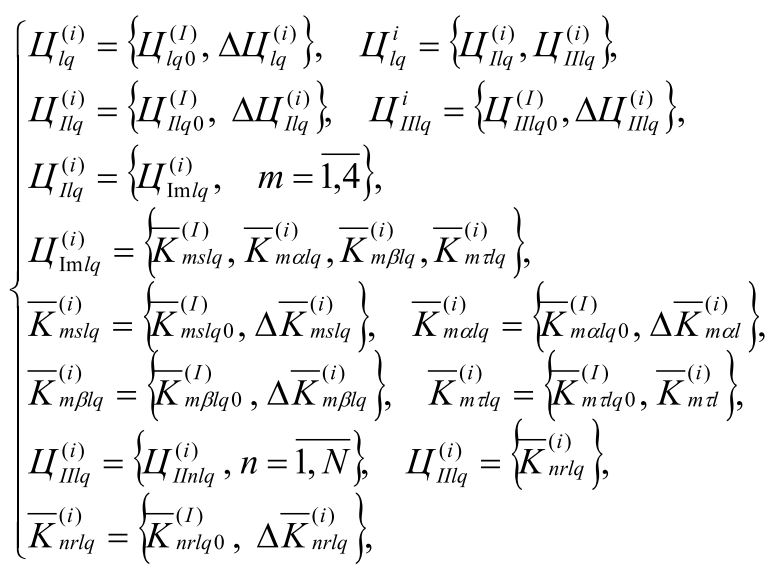




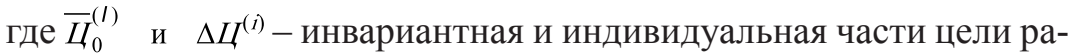
боты $i$-го сотрудника;

$\underline{m}$ и $n$ - компоненты качества работы сотрудника;

$\bar{K}_{0}^{(I)}$ и $\Delta K^{(i)}-$ нормативные показатели компонент качества работы $i$-го сотрудника;

Множество ресурсов, которые требуются для того, чтобы достигать $i$-ым сотрудником целей работы относительно $q$-го направления $\left\{\breve{q}_{q}^{(i)}-Y_{q}^{(i)}\right\}$ :

$$
{ }^{q} X_{l q}^{(i)}=\left\{C_{l q}^{(i)}, D_{l q}^{(i)} \Gamma_{l q}^{(i)}, O_{l q}^{(i)}, \dot{O}_{l q}^{(i)}, \ddot{I}_{l q}^{(i)}\right\}
$$

где $\mathrm{C}^{(i)}$ - содержание работы $i$-го сотрудника, представленное в плане:

$$
\begin{aligned}
& C_{l q}^{(i)}=\left\{C_{l q 0}^{(I)}, \quad \Delta C_{l q}^{(i)}\right\}, \quad C_{l q}^{(i)}=\left\{C_{I l q}^{(i)}, C_{I I l q}^{(i)}, C_{I I l q}^{(i)}\right\}, \\
& C_{I I I l q}^{(i)}=\left\{C_{I I I \mu l q}^{(i)}, \quad \mu=1, M\right\},
\end{aligned}
$$

$C_{\text {IIIiq }}^{(i)}$ - содержание моделей работы;

$D^{(i)}$ - совокупность средств оценки достижения $i$-ым сотрудником целей работы $\left\{L_{q}^{(i)}\right\}$ :

$$
D_{l q}^{(i)}=\left\{D_{l q 0}^{(i)}, \Delta D_{l q}^{(i)}\right\}, \quad D_{l q}^{(i)}=\left\{D_{l l q}^{(i)}, D_{\text {IIlq }}^{(i)}\right\}
$$

$\Gamma^{(i)}$ - совокупность характеристик работы і-го сотрудника:

$$
\begin{aligned}
& \Gamma_{l q}^{(i)}=\left\{\Gamma_{l q 0}^{(I)}, \Delta \Gamma_{l q}^{(i)}\right\}, \Gamma_{l q}^{(i)}=\left\{\Gamma_{I l q}^{(i)}, \Gamma_{I I l q}^{(I)}, \Gamma_{I I I q}^{(I)}\right\}, \\
& \Gamma_{I I l q}^{(i)}=\left\{\Psi_{\operatorname{lm} l q 0}^{(i)}, m=\overline{1,4}\right\}, \Gamma_{I l q}^{(i)}=\left\{\bigcup_{\mathrm{IImlq}}^{(i)}, m=\overline{1, N}\right\}, \\
& \Gamma_{I I I q}^{(i)}=\left\{\Psi_{I I H \mu q}^{(i)}, \mu=\overline{1, M}\right\}, \Gamma_{I l q}^{(i)}=\left\{\Gamma_{A l q}^{(i)}, \Gamma_{5 / q}^{(i)}\right\},
\end{aligned}
$$

Ч - количество часов на обеспечение работы,

$\Gamma_{A l q}^{(i)}$ и $\Gamma_{5 l q}^{(i)}-$ совокупность работ с непосредственным взаимодействием сотрудников и без непосредственного взаимодействия;

$O^{(i)}$ - множество ресурсов для работы $i$-го сотрудника:

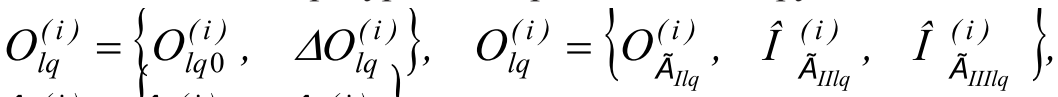

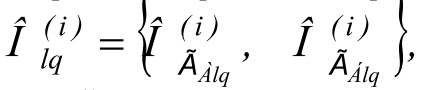

$$
\begin{aligned}
& \dot{O}_{l q}^{(i)}=\left\{\dot{O}_{l q}^{(I)}, \quad \Delta \dot{O}_{l q}^{(i)}\right\}, \quad \dot{O}_{l q}^{(i)}=\left\{\dot{O}_{a l q}^{(i)}, \quad \stackrel{a}{a}=\overline{1, E}\right\},
\end{aligned}
$$


$\boldsymbol{y}_{\mathrm{elq}}^{(i)}$ - совокупность значимых компонентов среды работы;

$\Pi^{(i)}-$ состав более опытных сотрудников, обеспечивающий взаимодействие с $i$-м сотрудником:

$$
\Pi_{l q}^{(i)}=\left\{\Pi_{p l q}^{(i)}, \quad p=\overline{1, P}\right\}, \quad \Pi_{\Pi p l q}^{(i)}=\left\{M_{\Pi_{p l q}}, \quad K B_{\Pi_{p l q}}\right\},
$$

$M_{\Pi_{p}}$ - мотивация $p$-го опытного сотрудника к своей деятельности,

$K B_{\Pi_{p}}-$ квалификация $p$-го опытного сотрудника.

Построение формализованной модель сравнительной оценки БПУ (1) $-Z_{q}^{(i)}$ происходит таким образом:

$$
\begin{aligned}
& Z_{l q}^{(i)}=H_{l q}^{(i)}\left(Y_{l q}^{(i)}, \quad \bigsqcup_{l q}^{(i)}, \quad B_{l q}^{(i)}, \quad D_{l q}^{(i)}, V_{l q}\right), \\
& Z_{l q}^{(i)}=\left\{\begin{array}{ll}
Z_{l l q}^{(i)}, \quad Z_{I l q}^{(i)}
\end{array}\right\},
\end{aligned}
$$

Здесь $Z^{(i)}$ - результаты оценки параметров сформированной у $i$-го сотрудника структуры качества работы; $B^{(i)}$ - множество субъектов, которые связаны с оценками; $D^{(i)}$ - множество ресурсов для проведения оценок оценки $[9,10] ; V$ - случайные факторы.

\section{Критерий эффективности модели}

Критерий эффективности работы транспортной системы необходимо рассматривать с точки зрения БПУ (1). Если проводить анализ детерминированным упрощенным способом, тогда он может быть записан так:

$$
J_{\perp l q}^{(i)}\left(L_{l q}^{(i)}\right)=\left\{f_{1 q q \gamma}\left(Y_{l q}^{(i)}, Ц_{l q}^{(i)}\right) \geq f_{1 l q \gamma}^{3 a \partial}\left(L_{l q}^{(i)}\right), \gamma=1, Y_{\perp l q}\left(Ц_{\partial \ddot{u}}^{(i)}\right)\right\} .
$$

При этом рассматриваются результаты $Z^{(i)(l-1) q}$, связанные с оценкой параметров наблюдаемых у $i$-го сотрудника характеристик качества функционирования.

Критерий эффективности с точки зрения своего содержания в ходе рассмотрения БПУ определяется в соответствии с тем, как в (6) будут выполняться все неравенства. Этот анализ проводится, когда есть фиксированные результаты по оценке качества функционирования $i$-го сотрудника. Такая оценка осуществляется с учетом информации по всем предыдущим шагам его работы. В указанных неравенствах: в виде функции $f_{1 l q \gamma}(\bullet)$ и числе неравенств $\gamma_{1 l q}\left(\bigsqcup_{l q}^{(i)}\right)$ происходит отражение компонентов соответствующих 
квалификационных требований для сотрудников по $l$-ому шагу работы. При этом анализируется $q$-е направление, когда рассматривается $Ц_{l q}^{(i)}$-я цель работы.

\section{Выводы}

При управлении функционированием транспортного предприятия можно использовать разработанную модель. Она связана с характеристиками работников, направлениями их работы. Критерий эффективности модели связан с квалификационными требованиями сотрудников.

Информация о конфликте интересов. Авторы заявляют об отсутствии конфликта интересов.

Информация о спонсорстве. Исследование не имело финансовой поддержки.

\section{Сиисок литературы}

1. Паневин Р.Ю., Преображенский Ю.П. Задачи оптимального управления многостадийными технологическими процессами // Вестник Воронежского института высоких технологий. 2010. № 6. С. 77-80.

2. Преображенский Ю.П. Информационные технологии, используемые в сфере менеджмента // Вестник Воронежского института высоких технологий. 2018. № 2 (25). С. 43-46.

3. Ермолова В.В., Преображенский Ю.П. Методика построения семантической объектной модели // Вестник Воронежского института высоких технологий. 2012. № 9. С. 87-90.

4. Преображенский Ю.П., Коновалов В.М. Анализ подходов к формированию рекомендательных систем // Вестник Воронежского института высоких технологий. 2019. № 4 (31). С. 88-90.

5. Преображенский Ю.П., Коновалов В.М. О методах создания рекомендательных систем //Вестник Воронежского института высоких технологий. 2019. № 4 (31). С. 75-79.

6. Берман Н.Д., Белов А.М. Общественный транспорт и инновации // International Journal of Advanced Studies. 2019. Т. 9. № 2. С. 7-13. 
7. Шакиров А.А., Зарипова Р.С. Особенности моделирования логистических систем // International Journal of Advanced Studies. 2019. T. 9. № 4. C. 27-31.

8. Сапожникова С.М. Корпоративное управление в железнодорожном транспорте // International Journal of Advanced Studies. 2019. Т. 9. № 4. С. 19-42.

9. Лысанов Д.М., Бикмухаметова Л.Т. Анализ показателей качества и конкурентоспособности оборудования // International Journal of Advanced Studies. 2018. T. 8. № 4-2. С. 50-55.

10. Преображенский Ю.П. Построение складской структуры предприятия // В сборнике: Молодежь и системная модернизация страны Сборник научных статей 3-й Международной научной конференции студентов и молодых ученых. В 4-х томах. Ответственный редактор А.А. Горохов. 2018. С. 286-290.

\section{References}

1. Panevin R.Yu., Preobrazhenskij Yu.P. Zadachi optimal'nogo upravleniya mnogostadijnymi tekhnologicheskimi processami // Vestnik Voronezhskogo instituta vysokih tekhnologij. 2010. № 6. S. 77-80.

2. Preobrazhenskij Yu.P. Informacionnye tekhnologii, ispol'zuemye v sfere menedzhmenta // Vestnik Voronezhskogo instituta vysokih tekhnologij. 2018. № 2 (25). S. 43-46.

3. Ermolova V.V., Preobrazhenskij Yu.P. Metodika postroeniya semanticheskoj ob»ektnoj modeli // Vestnik Voronezhskogo instituta vysokih tekhnologij. 2012. № 9. S. 87-90.

4. Preobrazhenskij Yu.P., Konovalov V.M. Analiz podhodov k formirovaniyu rekomendatel'nyh sistem // Vestnik Voronezhskogo instituta vysokih tekhnologij. 2019. № 4 (31). S. 88-90.

5. Preobrazhenskij YU.P., Konovalov V.M. O metodah sozdaniya rekomendatel'nyh sistem //Vestnik Voronezhskogo instituta vysokih tekhnologij. 2019. № 4 (31). S. 75-79.

6. Berman N.D., Belov A.M. Obshchestvennyj transport i innovacii // International Journal of Advanced Studies. 2019. T. 9. № 2. S. 7-13. 
7. Shakirov A.A., Zaripova R.S. Osobennosti modelirovaniya logisticheskih sistem // International Journal of Advanced Studies. 2019. T. 9. № 4. S. 27-31.

8. Sapozhnikova S.M. Korporativnoe upravlenie v zheleznodorozhnom transporte // International Journal of Advanced Studies. 2019. T. 9. № 4. S. $19-42$.

9. Lysanov D.M., Bikmuhametova L.T. Analiz pokazatelej kachestva i konkurentosposobnosti oborudovaniya // International Journal of Advanced Studies. 2018. T. 8. № 4-2. S. 50-55.

10. Preobrazhenskij Yu.P. Postroenie skladskoj struktury predpriyatiya // V sbornike: Molodezh' i sistemnaya modernizaciya strany Sbornik nauchnyh statej 3-j Mezhdunarodnoj nauchnoj konferencii studentov i molodyh uchenyh. V 4-h tomah. Otvetstvennyj redaktor A.A. Gorohov. 2018. S. 286-290.

\section{ДАННЫЕ ОБ АВТОРАХ}

Львович Яков Евсеевич, профессор, доктор технических наук, профессор

Воронежский государственный технический университет ул. 20 лет Октября, 84, Воронеж, 394006, Российская Федерачия

Komkovvivt@yandex.ru

Преображенский Андрей Петрович, профессор, доктор технических наук, доцент

Воронежский институт высоких технологий ул. Ленина, 73а, Воронеж, 394043, Российская Федераџия

Чопоров Олег Николаевич, профессор, доктор технических наук, профессор

Воронежский государственный технический университет ул. 20 лет Октября, 84, Воронеж, 394006, Российская Федерачия

Komkovvivt@yandex.ru 


\section{DATA ABOUT THE AUTHORS}

Lvovich Yakov Yevseevich, Doctor of Technical Sciences, Professor Voronezh State Technical University

84, 20 years of October Str., Voronezh, 394006, Russian Federation

Komkovvivt@yandex.ru

ORCID: 0000-0002-7051-3763

Preobrazhenskiy Andrey Petrovich, Professor, Doctor of Technical Sciences, Associate Professor

Voronezh Institute of High Technologies

73a, Lenin Str., Voronezh, 394043, Russian Federation

Komkovvivt@yandex.ru

ORCID: 0000-0002-6911-8053

Choporov Oleg Nikolaevich, Doctor of Technical Sciences, Professor Voronezh State Technical University

84, 20 years of October Str., Voronezh, 394006, Russian Federation

Komkovvivt@yandex.ru

ORCID: 0000-0002-3176-499X 